\title{
Examining the barriers of influenza vaccine hesitancy in persons with dementia: a literature review
}

\author{
Bahar Ashtarieh $^{1}$ (D) Magda Grabkowski ${ }^{1} \cdot$ Emma Bartfay $^{1} \cdot$ Winnie Sun $^{1}$
}

Received: 1 April 2021 / Accepted: 9 July 2021 / Published online: 28 July 2021

(c) The Author(s), under exclusive licence to Springer Nature Switzerland AG 2021

\begin{abstract}
Background/aims Despite the proven benefits of vaccine prevention and the continuous development of this important public health measure, vaccine hesitancy is among the top ten threats to global health according to the World Health Organization in 2019. Those who contract the influenza virus will typically develop a mild illness. However, for populations at a higher risk, including those diagnosed with dementia, influenza is proven to be more detrimental. Persons with dementia (PWD) face a serious threat to influenza on an annual basis, and vaccine hesitancy may further potentiate this risk. The purpose of this paper is to explore and understand the barriers PWD face in receiving the influenza vaccine.

Methods A literature review was conducted to examine the barriers that prevent PWD from seeking out the annual influenza vaccine. A thematic analysis provided an understanding of the overarching relationships found among the influences between the identified themes.

Results Three main influences were identified: (1) intra-personal, (2) inter-personal, and (3) extra-personal, with each influence containing several subsequent sub-themes.

Discussion/conclusion The literature review found that there is a lack of research involving this high-risk population and the barriers they face, especially within the extra-personal influences. Further research is required to understand how each barrier relates and connects with each other. Understanding this connection will aid public health organizations in decreasing the threat of vaccine hesitancy, thus decreasing the incidents of preventable deaths.
\end{abstract}

Keywords Influenza vaccine $\cdot$ Vaccine uptake $\cdot$ Vaccine hesitancy $\cdot$ Dementia $\cdot$ Barriers $\cdot$ Public health

\section{Introduction}

The Center for Disease Control and Prevention (CDC) describes that most individuals affected by influenza will have mild illness [1]. However, for a population that is considered high risk, influenza prevalence is higher, and complications are more detrimental. Cognitive impairment as result of dementia may present major barriers for the individual to fight against infection, thus resulting in an increased likelihood to develop serious complications [2]. In fact, dementia is an independent risk factor for influenza complications and can be especially dangerous as early symptoms of influenza are difficult to recognize in persons with dementia (PWD) [3]. As a result, PWD are hospitalized more frequently and

Winnie Sun

winnie.sun@ontariotechu.ca

1 Faculty of Health Sciences, Ontario Tech University, 2000 Simcoe Street North, Oshawa, ON L1G 0C5, Canada for longer durations [4]. Consequently, due to the higher morbidity rates, individuals with chronic neurological diseases such as dementia also face higher mortality rates. This population is 40 times more likely to die from complications due to influenza than those who are not affected by neurological conditions [2].

The best influenza prevention strategy is receiving the annual vaccine. In Canada, during the 2017/2018 influenza season, the overall adult vaccine coverage was $38.3 \%$, while coverage for the high-risk population of seniors over 65 years of age was $70.7 \%$, which is below the national coverage goal of $80 \%$ [5]. Even more specifically, a Canadian study about influenza vaccine uptake showed that approximately $55 \%$ of the sample population living with dementia had received the flu vaccine [6]. It is an alarming result as close to half of the participating PWD population had not received the influenza vaccine, despite this population having a greater risk of contracting influenza and its deadly complications [6]. 
A systematic review of influenza vaccine efficacy for older persons concluded that the influenza vaccine reduces the risk for pneumonia, hospitalization, and death in older persons if the strain of the vaccine is the same or similar to the influenza epidemic strain [7]. The review also discussed that all persons older than 65 should have the influenza vaccine as a part of their care plan, and this requires involvement from informal caregivers, physicians, and public health organizations. Despite these factors posing as motivations for PWD to receive influenza vaccine, uptake among this population is inferior.

\section{Objectives}

The objection or rejection of vaccines, despite their availability, is known as vaccine hesitancy [8]. It is evident that PWD have an increased risk for influenza complications. This, coupled with the lack of vaccine uptake specifically in this population, makes the seemingly harmless annual influenza season a deadly threat to PWD. To combat this public health issue of vaccine hesitancy, it is essential to identify and understand the various barriers that this population faces when it comes to accessing the influenza vaccine. The purpose of this literature review is to explore the barriers PWD face in receiving the influenza vaccine.

\section{Methods}

A literature review was conducted to examine the barriers PWD face in receiving the influenza vaccination. This included barriers that may be a result of care from informal caregivers (ICs) and health care providers (HCPs). The three concepts used for the initial search were (1) vaccines, (2) dementia, and (3) barriers. Within each concept, there were different keywords used. The vaccine concept used vaccine*, immuniz*, or immunis*; the dementia concept used Alzheimer* or dementia; and the barriers concept used access*, barrier*, or convenien*. A variety of databases were accessed for the search, primarily PubMed, CINAHL, the Ontario Tech University database, and Google Scholar. Each database search consisted of using the same keywords and pairing either two or three of the concepts together. On CINAHL, for example, pairing all three concepts yielded only 22 results, so a broader search of pairing two concepts at the same time was used. Titles and abstracts were first screened for relevance to the research question, and only articles published in English were used. Reference pages from peer-reviewed articles were scanned for relevant resources. Overall, 24 resources were used to conduct a thematic analysis (See Table 1).

\section{Results}

Altogether, we classified the themes among three major influences: (1) intra-personal, (2) inter-personal, and (3) extra-personal. A summary and relationship between these three themes and their sub-themes is presented in Fig. 1. Table 1 presents the details of the contributions from each study, while Table 2 presents a summary chart of each sub-theme.

Intra-personal influences include themes that directly relate to the PWD, including dementia-related symptoms, personal factors, and culture and race. Dementia-related symptoms relate to the cognitive and motor decline that PWD experience. As a result of this decline, PWD may have difficulty accessing, understanding, and adhering to the influenza vaccine [9-13]. Personal factors of PWD, specifically marital/relationship status, was a common theme found among the literature $[14,15]$. Culture and ethnicity play a role in this public health crisis as cultural traditions, beliefs, and superstitions may influence decisions made by PWD, HCPs, and ICs [16-19]. Some sources discussed how past public health controversies involving minorities continue to create distrust, thus resulting in low influenza vaccine uptake in these specific minority populations $[17,18]$.

Inter-personal influences extend beyond the PWD, instead involving those who directly aid in their care, primarily IC and HCP. Literature suggests that caregiver distress may influence the decision to seek out a vaccination for the PWD in their care [20, 21]. In a similar way, the financial burden that some ICs experience can influence the accessibility aspect of vaccines [4]. HCP impact refers to all the factors a HCP may possess that can alter the likelihood of a PWD receiving the vaccine. Personal factors for HCPs refer to gender, lifestyle, and immunization status [22, 23]. Additionally, the level of trust between HCP, IC, and PWD was found to be a contributing factor. Finally, the beliefs of complementary and alternative medicine (CAM) providers play an important role in accessing vaccination as they often offer vaccine advice to patients and families [24].

The final major influence is extra-personal, which involves everything beyond those in direct care of the PWD. A common environmental influence was accommodation and accessibility of the vaccine based on living situations $[25,26]$. Our research also indicated that media influence was one of the driving factors in vaccine hesitancy [27-31]. With online and social media usage being at an all-time high, finding misinformation and anti-vaccination movements can be easy, thus negatively changing the perception of influenza vaccinations. 







$$
11
$$




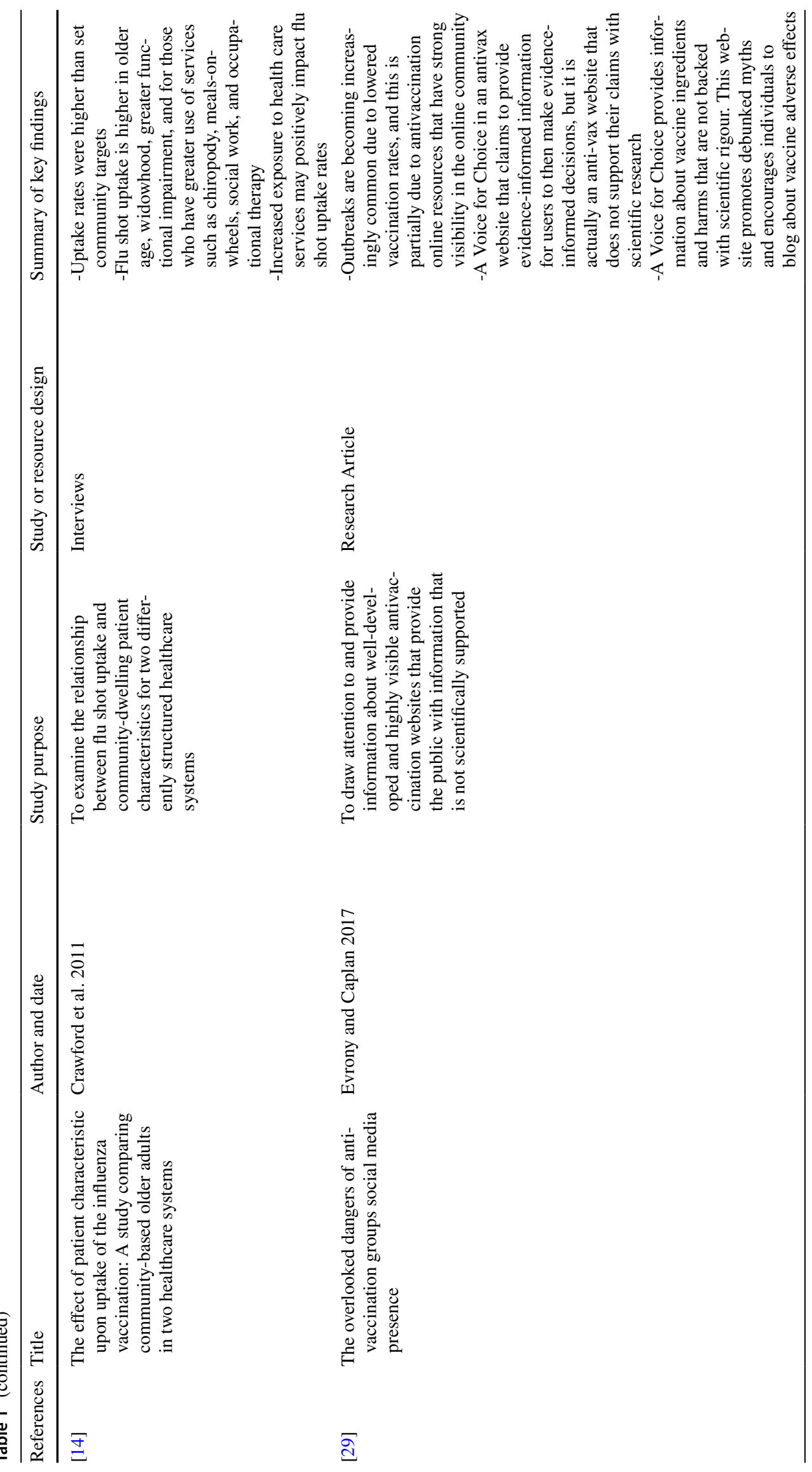




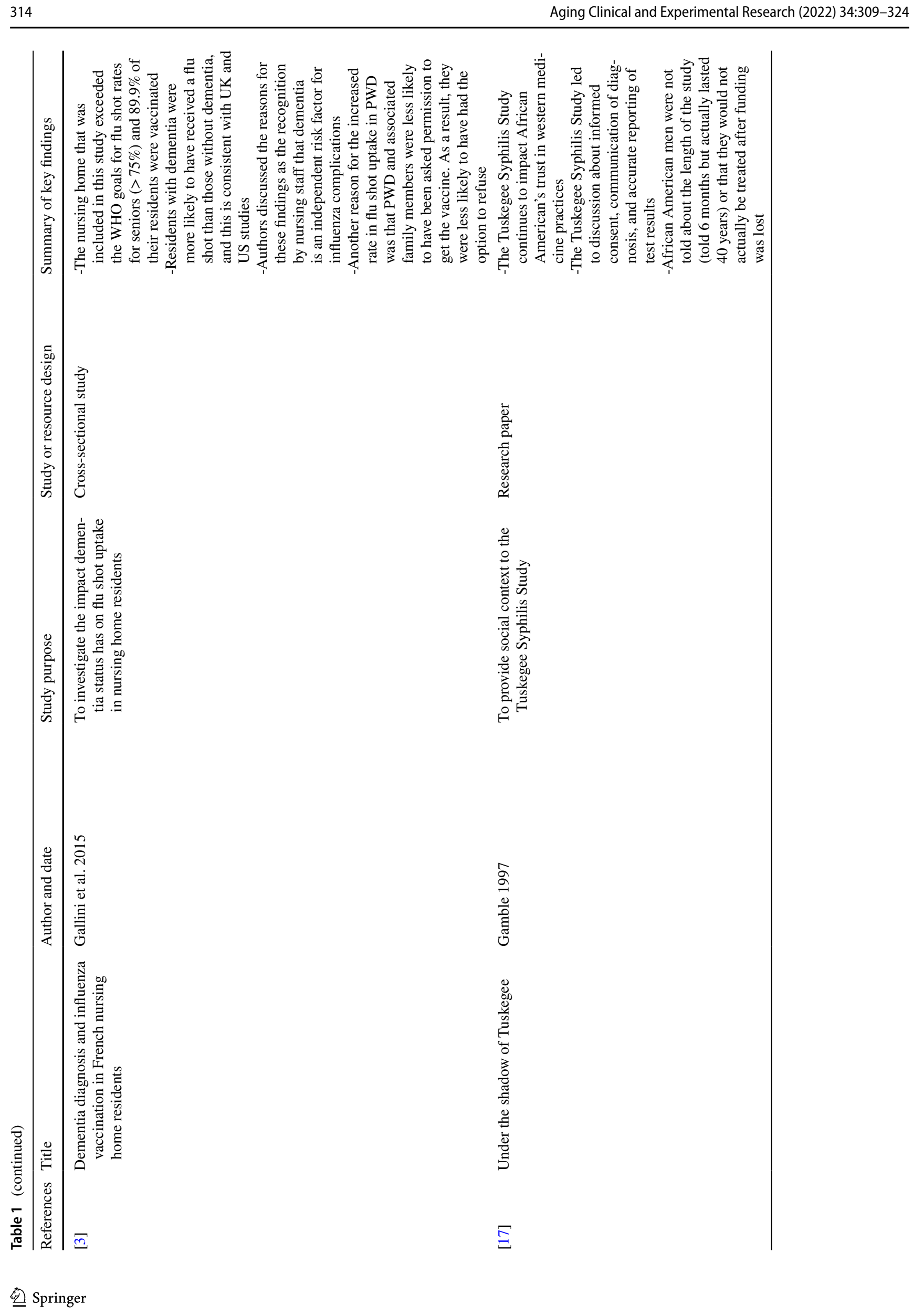




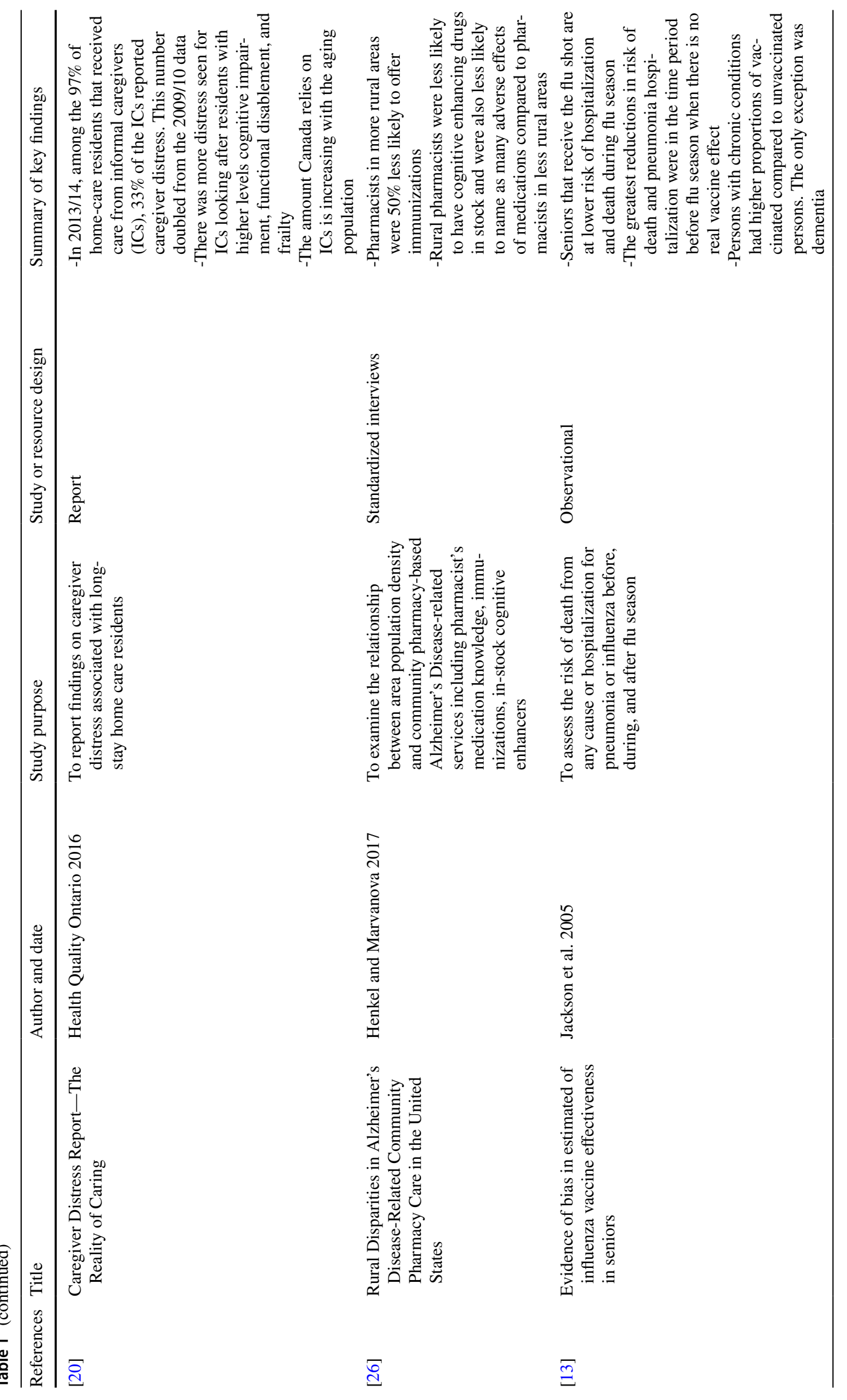




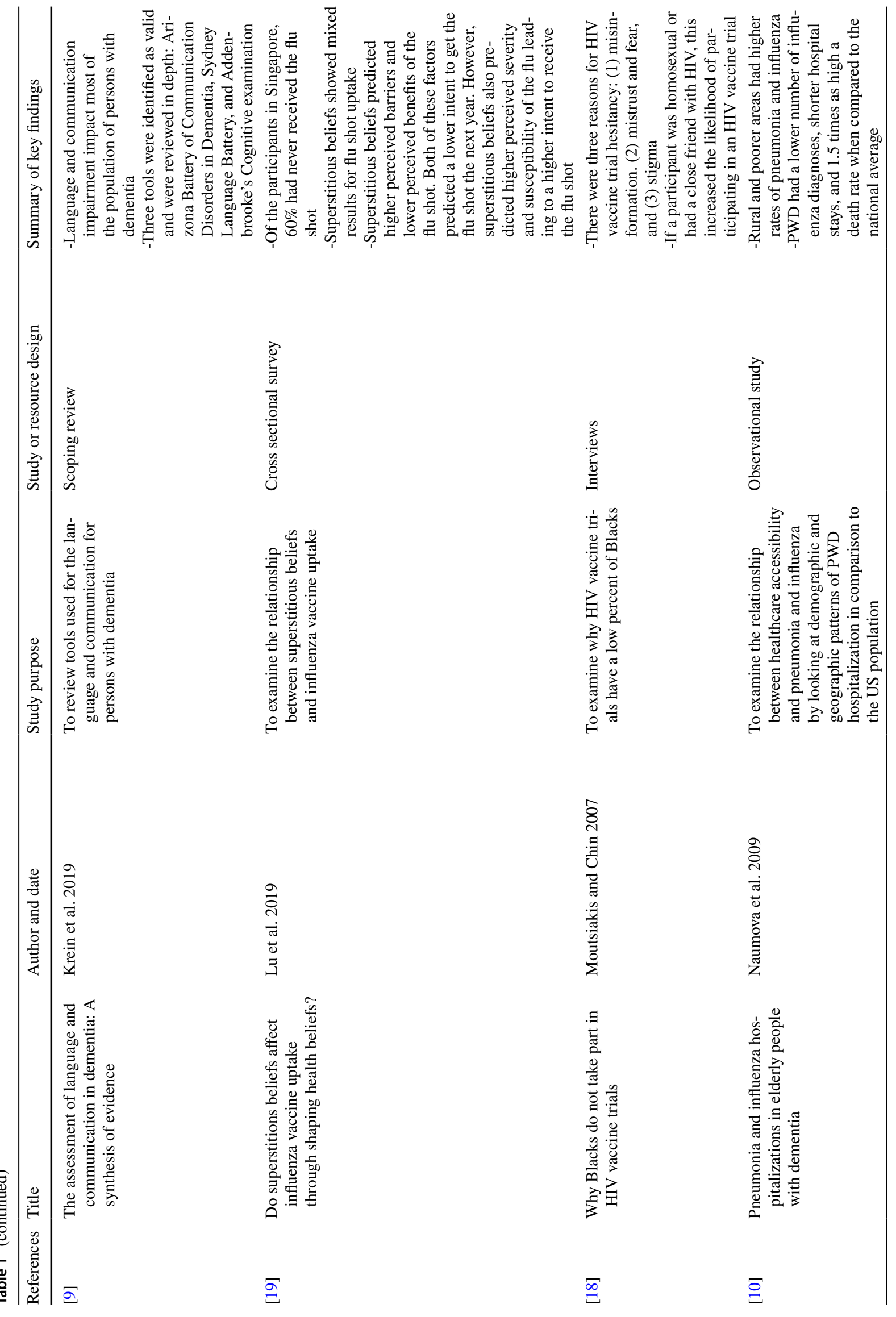




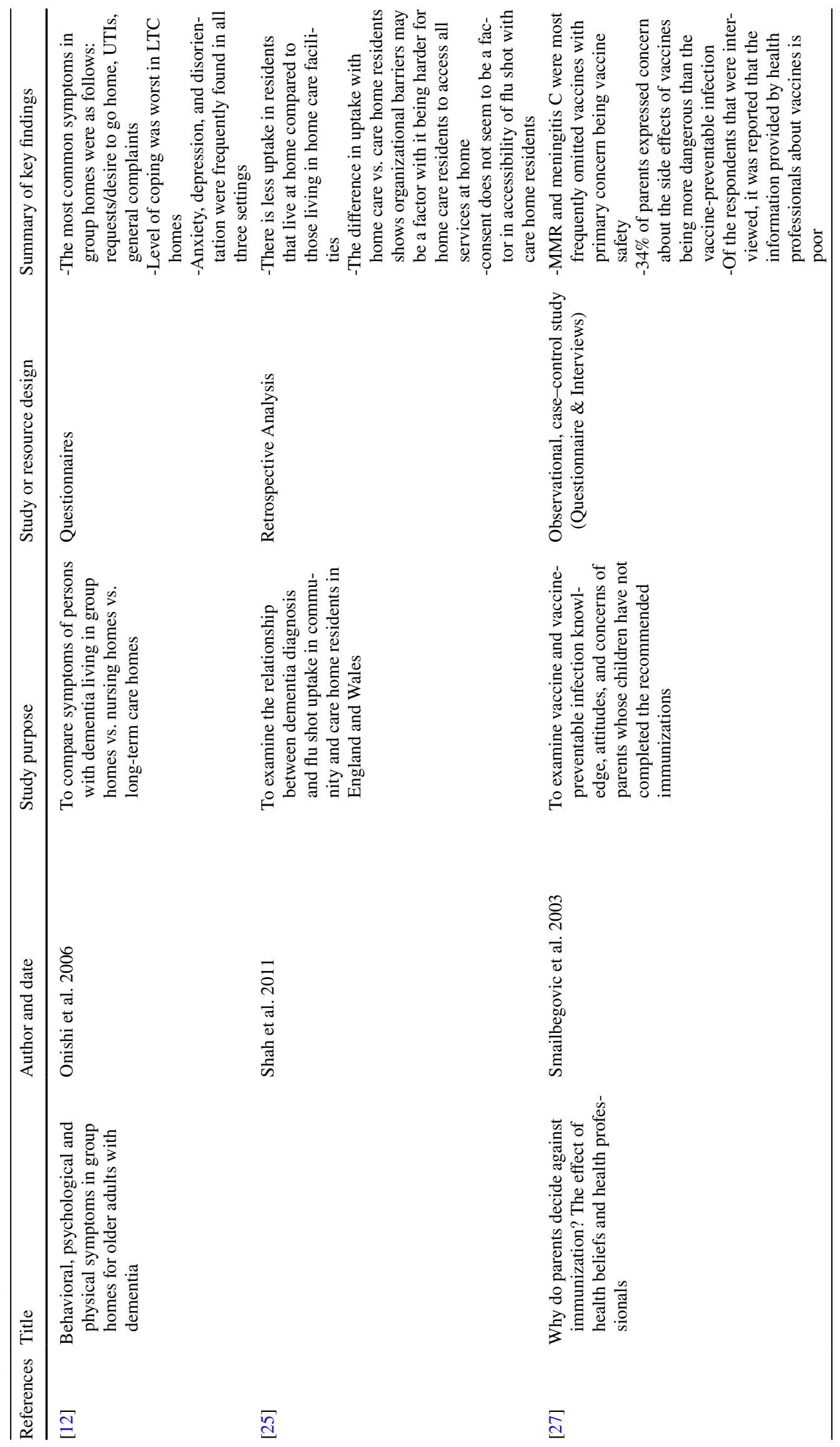




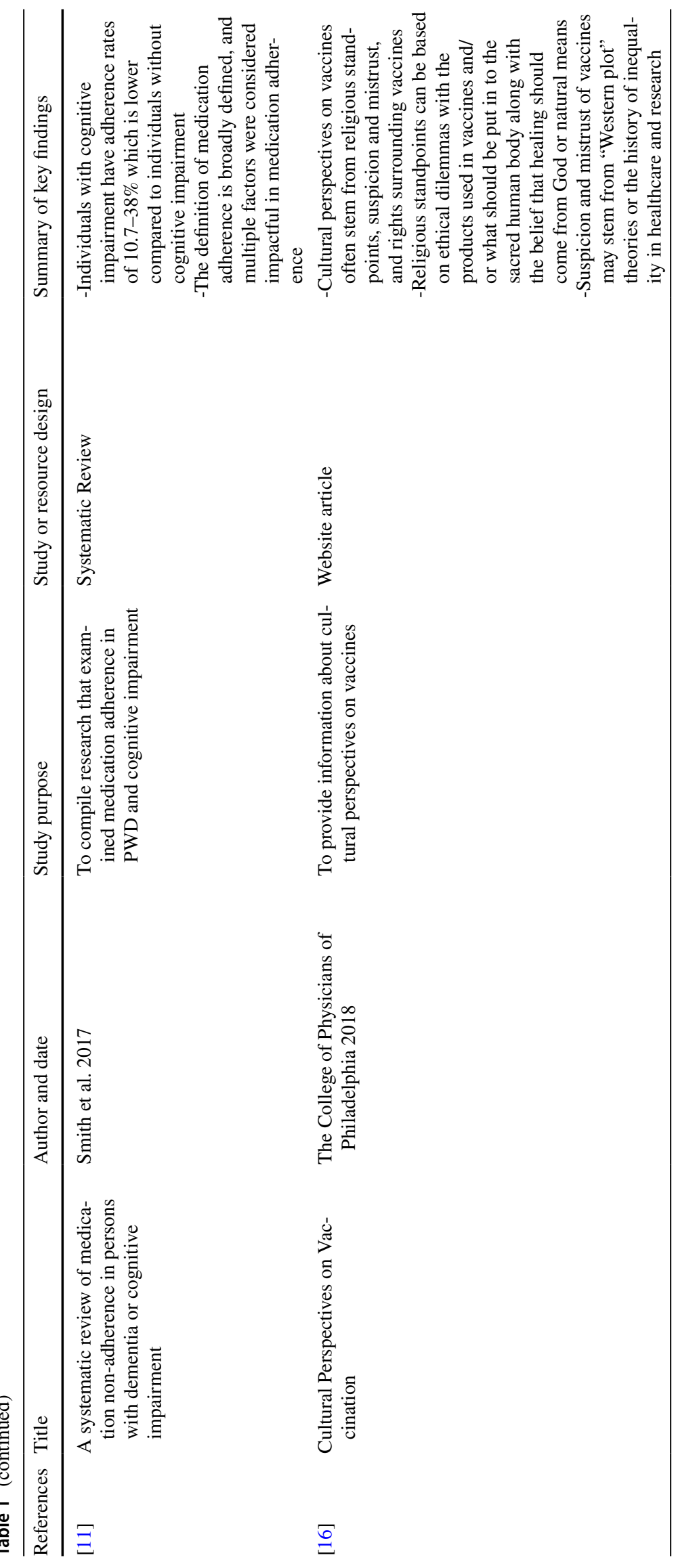




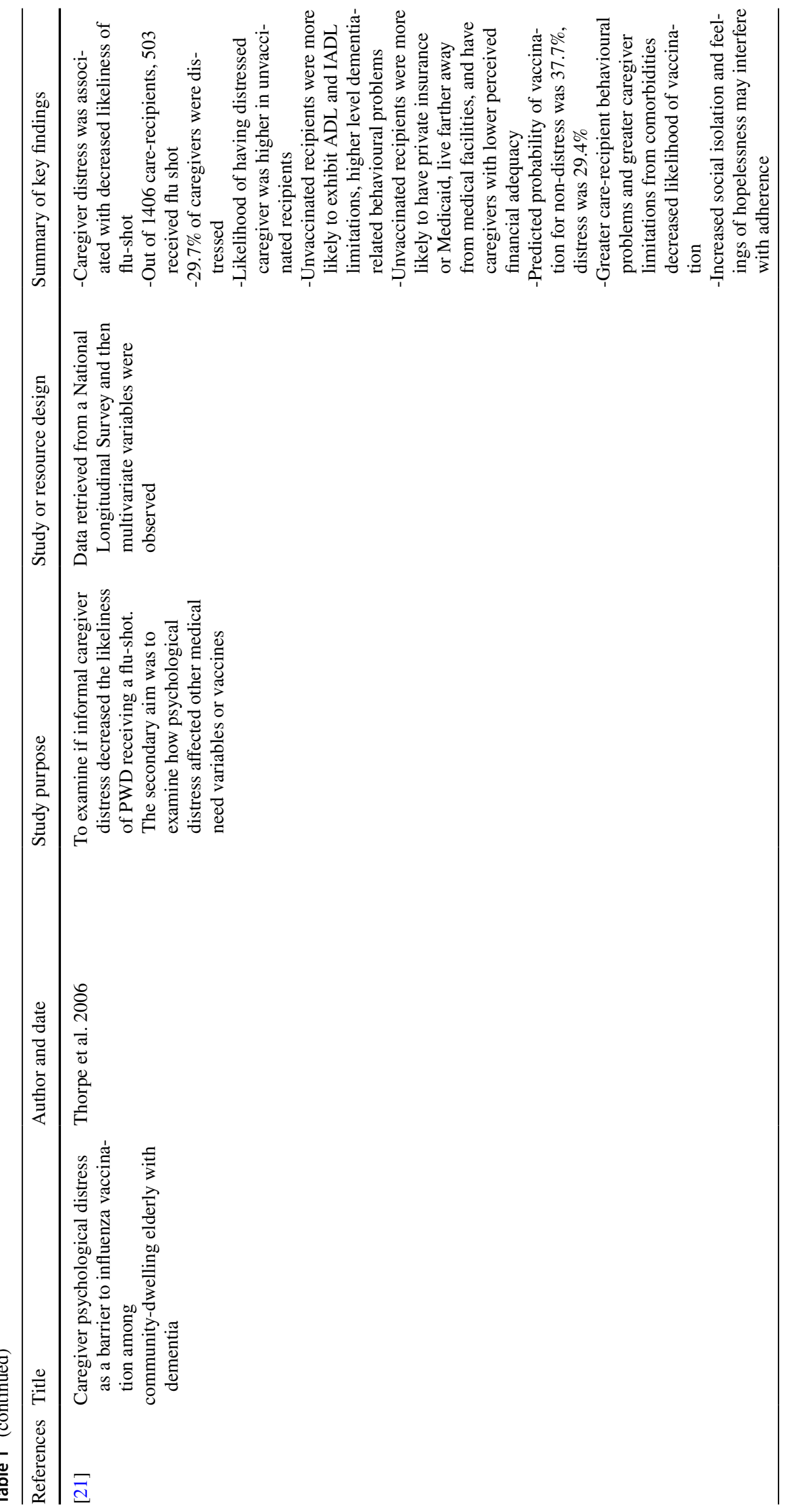




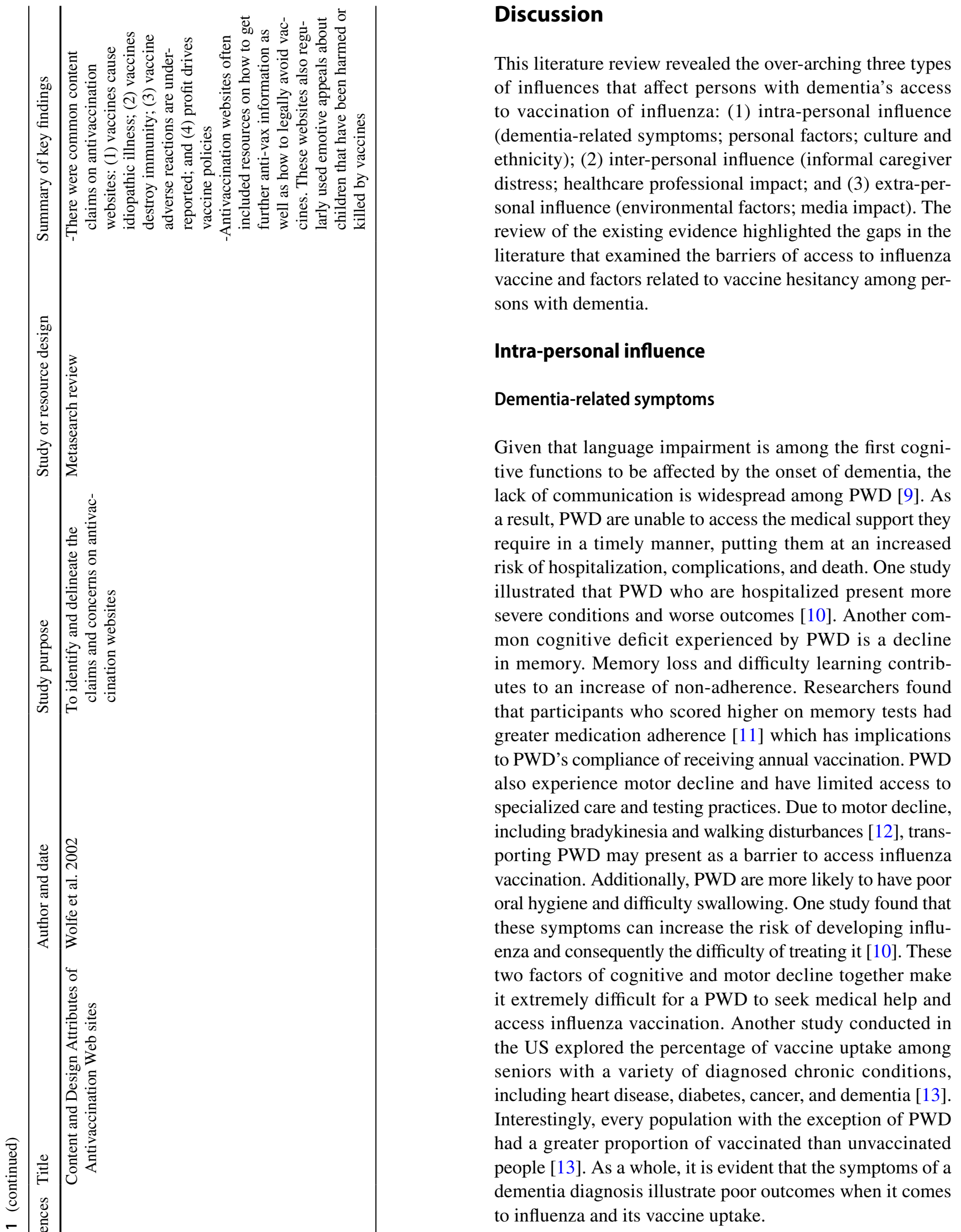

Given that language impairment is among the first cognilack of communication is widespread among PWD [9]. As a result, PWD are unable to access the medical support they require in a timely manner, putting them at an increased One study more ther comon cognitive deficit experienced by PWD is a decline memory. Memory loss and difficulty learning contribto an increase of non-adherence. Researchers found that participants who scored higher on memory tests had reater medication adherence [11] which has implications PWD's compliance of receiving annual vaccination. PWD lso experience motor decline and have limited access to secialized care and testing practices. Due to motor decline, ncluding bradykinesia and walking disturbances [12], transporting PWD may present as a barrier to access influenza vaccination. Additionally, PWD are more likely to have poor oral hygiene and difficulty swallowing. One study found that hese symptoms can increase the risk of developing influenza and consequently the difficulty of treating it [10]. These wo factors of cognitive and motor decline together make t extremely difficult for a PWD to seek medical help and access influenza vaccination. Another study conducted in he US explored the percentage of vaccine uptake among seniors with a variety of diagnosed chronic conditions, including heart disease, diabetes, cancer, and dementia [13]. Interestingly, every population with the exception of PWD had a greater proportion of vaccinated than unvaccinated people [13]. As a whole, it is evident that the symptoms of a to influenza and its vaccine uptake. 


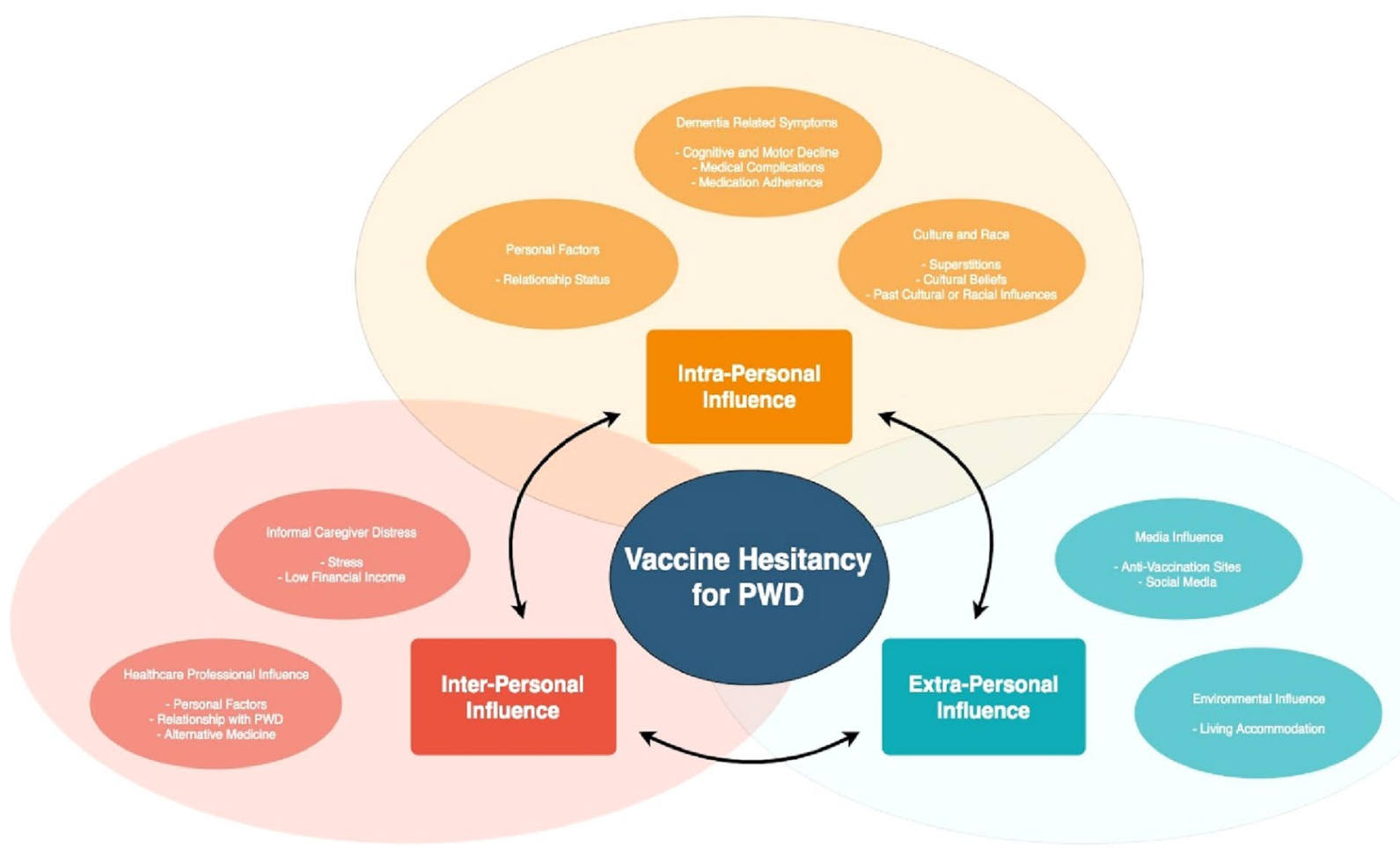

Fig. 1 Summary and relationship between the three major influences identified, along with their sub-themes

Table 2 Summary of findings, including factors of each theme, as well as the negative outcomes that prevent PWD from actively seeking out an influenza vaccine

\begin{tabular}{lll}
\hline Summary of findings & & \\
\hline Themes & Factors & Outcomes \\
\hline Dementia-related symptoms & Cognitive and motor decline & Medical complications \\
Personal factors & Relationship status & $\begin{array}{l}\text { Medreased accessibility } \\
\text { Decreased accessibility }\end{array}$ \\
& & Potential misinformation \\
Culture and ethnicity & Superstitions & Lack of motivation \\
& Cultural beliefs & Medication adherence \\
& Past cultural or ethnic influences & Lack of trust in vaccine and healthcare providers \\
Healthcare professional (HCP) impact & Personal factors & Lack of trust in HCP \\
& Relationship status with PWD and/or IC & Lack of positive influence \\
& Alternative medicine beliefs & Misinformation \\
Informal caregiver (IC) distress & Stress & Lack of motivation \\
& Low financial income & Decreased accessibility \\
& & Misinformation \\
Environmental factors & Living accommodation & $\begin{array}{l}\text { Decreased accessibility to healthcare services } \\
\text { Potential misinformation }\end{array}$ \\
Media impact & Anti-vaccination sites & Misinformation \\
& Social media & Lack of trust in vaccine and healthcare providers
\end{tabular}




\section{Personal factors}

Relationship status, specifically widowhood, was another factor found to have an influence on the likelihood of an older adult seeking out the influenza vaccination. Researchers theorized that due to the increased vulnerability of widows, this population may have an increased likelihood of vaccination [14]. On the other hand, it may be possible that due to the increased caregiving and monitoring of PWD, family caregivers often have the assumptions that PWD may not necessarily require to receive the influenza vaccine. Another study found that participants who lived with others were more likely to get the vaccine than those who were living alone [15]. A limitation of this study was that the participants included were recruited randomly from the general populations, which had different characteristics than persons with dementia. Due to these mixed results, it is essential to conduct further research to truly understand how relationship status may affect influenza vaccine uptake. Regardless, it is evident from the existing literature that personal factors play a role in influencing PWD's access to influenza vaccination.

\section{Culture and ethnicity}

Cultural beliefs may impact vaccine uptake. These cultural beliefs are typically based around the usage of human tissue cells in vaccines, with the belief that the body is sacred and should not receive chemicals, blood, or tissues from animals; as well as the idea that God or natural means will heal the body [16]. Further, the distrust of modern medicine can stem from historical and social inequality and mistrust. This is displayed by the African Americans' distrust in medical treatments, including vaccination, after being denied appropriate treatment during the Tuskegee Syphilis study [17]. Another study also indicated that Black populations were more likely to refuse the vaccine, expressing concerns about vaccine safety and efficacy [18].

Lu et al. [19] studied how superstitious beliefs may specifically impact influenza vaccine uptake in Singapore. It was found that superstitions do have an impact on flu vaccine uptake. However, the impacts were mixed. Superstitious beliefs led to a predicted lower uptake because of higher perceived barriers and lower perceived benefits of vaccines. In contrast, some superstitious beliefs led to a higher uptake because of higher perceived susceptibility and severity of influenza. The impact of intra-personal factors has implications about persons with dementia's vaccine uptake, and future health education about vaccination should take into considerations about factors related to personal beliefs, cultural background, and ethnicity.

\section{Inter-personal influence}

\section{Informal caregiver distress}

Health Quality Ontario reports an ongoing increase in caregiver distress [20], and this may impact vaccine uptake. Caregiver distress refers to the stress and exhaustion some ICs feel due to the responsibility of caring for a PWD. Thorpe et al. examined caregiver psychological distress as a barrier to influenza vaccination uptake in communitydwelling PWD [21]. From a sample of 1406 PWD who were male veterans, and their corresponding female IC, it was found that caregiver distress was associated with decreased likeliness of the PWD receiving the influenza vaccine. The likelihood of having a distressed caregiver was higher in unvaccinated care recipients. Unvaccinated recipients were more likely to exhibit limitations in their activities of daily living (ADLs) and instrumental activities of daily living (IADLs), and had higher level dementia-related behavioural problems. Additionally, unvaccinated recipients were more likely to have private insurance or use Medicaid, suggesting that financial barrier play an important role in vaccine uptake. Unvaccinated recipients were more likely to have caregivers with lower perceived financial adequacy and who lived farther away from medical facilities [4].

\section{Healthcare professional impact}

Healthcare providers, especially family physicians, are typically the first link or connection to the healthcare system. Several different factors were found to influence influenza vaccine uptake, including HCP's personal characteristics and the relationship between the HCP and their patients. A study conducted in Israel found that patients were more likely to be immunized if their physician was also immunized [22]. Female doctors were more likely to be vaccinated than male doctors, which coincides with the finding that female doctors give stronger recommendations for vaccination than their male counterparts. In addition to physician's gender, their background and lifestyle also contributed to the findings [22]. Physicians who were educated in Western Europe or America, as well as physicians who exercised were more likely to be vaccinated. Furthermore, physicians who had greater knowledge about the complex risk factors associated with influenza, such as the high risk of lower respiratory infections and mortality rates among PWD, were more likely to make stronger recommendations to PWD for getting the vaccine, while being more reluctant to accept refusal [23].

Bean and Catania [24] examined CAM providers' beliefs regarding vaccinations since these providers may play an influential role in vaccine uptake for their clients. For the CAM providers that endorsed vaccinations, their reasoning was likely to fall within two themes: "vaccines prevent 
illness" and "herd immunity". In contrast, for the CAM providers that did not endorse vaccines, their reasoning was likely to fall within three themes: "natural is best", "innate intelligence", and "the fragile immune system". The nonendorsers focus was on providing health for the person rather than health for the population, and this emphasis has implications about the potential vaccine uptake among persons with dementia, who relied heavily on the advice from healthcare professionals to access vaccinations.

\section{Extra-personal influence}

\section{Environmental factors}

One of the more common environmental factors contributing to vaccine uptake among PWD was living accommodation. PWD were less likely than their counterparts to get the vaccine if they lived alone in their community. Researchers suggested that this result can be attributed to poor access of preventative care by PWD living in their own homes [25]. For those living in rural neighbourhoods, PWD may face even greater difficulty in accessing care and vaccination. Henkel and Marvanova [26] found that pharmacies in more rural areas are about $50 \%$ less likely to offer immunizations compared to less rural areas. Furthermore, Shah et al. [25] found that dementia does have an impact on influenza vaccine uptake in PWD who live in their homes. There is less vaccine uptake among PWD who live at home compared to those living in congregate and institutionalized settings. On the other hand, PWD were more likely to get the vaccine while living in a nursing or residential home compared to those without dementia [25]. Research found that $89.6 \%$ of PWD living in French nursing homes had the influenza vaccine administered, which is higher than the recommendation made by the WHO for that year ( $\geq 75 \%$ of older persons) [3]. One of the reasons the authors provided for this positive finding is that nursing home staff recognize that dementia is an independent risk factor for influenza complications.

\section{Media impact}

Media can impact an individual's belief as he/she seeks out information from a variety of external sources. It was proposed that the strongest predictor of immunization uptake for children was parental attitude [27], so it is essential to have evidence-informed information for those who make decisions about vaccination. A study examining MMR (Mumps, Measles, Rubella) vaccine uptake reported that parents that have confidence in vaccination effectiveness are more likely to have their children vaccinated. However, respondents felt that the information they received from HCPs about vaccinations was biased, and vaccination safety information was not forthcoming [27]. If individuals feel that the information they are receiving from HCPs is biased, they are more likely to seek information elsewhere. There are dangers associated with this approach. Specifically, not all internet resources are credible or reliable for an individual to use when making informed decisions, for example $A$ Voice for Choice. A Voice for Choice is a non-profit website that has a strong online following, and it claims to be promotive of evidenceinformed decision-making. However, there is a clear intention of promoting anti-vaccination [28, 29]. Davies et al. [30] investigated the likelihood of finding anti-vaccination sites when people search "immunization" or "vaccination". They found that $43 \%$ of resultant websites were related to anti-vaccination, and this result shows that there is a high probability that individuals seeking information about vaccinations are likely to discover anti-vaccination information. These anti-vax websites most commonly use emotional appeal to display the idea that vaccines cause idiopathic illness and destroy immunity. They also commonly impact vaccine confidence by sharing those adverse reactions to vaccines are underreported and that vaccination policies are motivated by profit [31]. The analogy of MMR vaccination and parental attitudes has implications for understanding persons of dementia's vaccine hesitancy because they often rely on caregivers to make decisions on their behalf.

\section{Study limitations}

There is a lack of existing literature that examines the barriers PWD face in receiving the influenza vaccine. While this topic is under-studied, our current literature review was able to highlight some of the factors that may impact the vaccine uptake among dementia populations. However, this literature review has limitations that need to be acknowledged. Most evidently, some literature being examined may not related directly to the study topic, but rather it provides implications to enhance our understanding about the potential factors that impact PWD's influenza vaccine uptake. A systematic review may potentially offer a more comprehensive approach to understanding the phenomenon of interest, as well as providing an appraisal of the included literature to assess the strength and quality of the available evidence.

\section{Conclusion}

Despite the categorization into the three over-arching themes, it is important to underscore that each theme, subtheme, and stakeholder group is interconnected, and altogether influencing access to influenza vaccination among PWD. Based on this literature review, certain sub-themes may need to be examined more closely to help advance our understanding and implement effective strategies to improve vaccine uptake among PWD. Today, social media 
and online sources play a major role in contributing to vaccine hesitancy. Future research needs to focus on examining the facilitating factors to overcome the challenges of this important public health issue.

Funding Partial financial support was received from Social Sciences and Humanities Research Council of Canada (SSHRC) Small Grant Program.

Availability of data and material The data supporting the findings of this literature review are available within the article and/or supplementary materials. Additional data are available on request from the corresponding author, [WS].

Code availability Not applicable.

\section{Declarations}

Conflict of interest The authors declare that they have no conflict of interest.

Ethics approval Not required (not a human study).

Consent to participate Not applicable.

Consent for publication All named authors have contributed to the creation of the submission and grant Aging Clinical and Experimental Research permission to review and (if selected) publish their work.

\section{References}

1. Centers for Disease Control and Prevention (2018) Who is at high risk for flu complications. U.S. Department of Health \& Human Services

2. Alzheimer's Society UK (2018) Why everyone affected by Dementia should have a flu jab. Alzheimer's Society UK

3. Gallini A, Gardette V, Lapeyre-Mestre M et al (2015) Dementia diagnosis and influenza vaccination in French nursing home residents. J Am Geriatr Soc 63:1256-1258

4. Canadian Institute for Health Information (2019) Dementia in Canada: Summary. Canadian Institute for Health Information

5. Government of Canada (2019) Vaccine uptake in Canadian adults 2019. Government of Canada

6. Campitelli MA, Rosella LC, Stukel TA et al (2010) Influenza vaccination and all-cause mortality in community-dwelling elderly in Ontario, Canada, a cohort study. Vaccine 29:240-246

7. Gross P, Hermogenes A, Sacks H et al (1996) The efficacy of influenza vaccine in elderly persons. A meta-analysis and review of the literature. Ann Intern Med 123:518-527

8. World Health Organization (2019) Ten threats to global health in 2019. https://www.who.int/emergencies/ten-threats-to-globalhealth-in-2019. Accessed 20 Jan 2020

9. Krein L, Jeon YH, Amberber AM et al (2019) The assessment of language and communication in dementia: a synthesis of evidence. Am J Geriatr Psychiatry 27:363-377

10. Naumova EN, Parisi SM, Castronovo D et al (2009) Pneumonia and influenza hospitalizations in elderly people with dementia. J Am Geriatr Soc 57:2192-2199
11. Smith D, Lovell J, Weller C et al (2017) A systematic review of medication nonadherence in persons with dementia or cognitive impairment. PLoS ONE 12(2):e0170651

12. Onishi J, Suzuki Y, Umegaki H et al (2006) Behavioral, psychological and physical symptoms in group homes for older adults with dementia. Int Psychogeriatr 18:75-86

13. Jackson LA, Jackson ML, Nelson JC et al (2006) Evidence of bias in estimates of influenza vaccine effectiveness in seniors. Int J Epidemiol 35:337-344

14. Crawford VLS, O'Hanlon A, McGee H (2011) The effect of patient characteristics upon uptake of the influenza vaccination: a study comparing community-based older adults in two healthcare systems. Age Ageing 40:35-41

15. Burns VE, Ring C, Carroll D (2005) Factors influencing influenza vaccination uptake in an elderly, community-based sample. Vaccine 23:3604-3608

16. The College of Physicians of Philadelphia (2018) Cultural perspectives on vaccination. The College of Physicians of Philadelphia

17. Gamble VN (1997) Under the Shadow of Tuskegee: African Americans and Health Care. Am J Public Health 87:1773-1778

18. Moutsiakis D, Chin P (2007) Why blacks do not take part in HIV vaccine trials. J Natl Med Assoc 99:254-257

19. Lu J, Luo M, Yee AZH et al (2019) Do superstitious beliefs affect influenza vaccine uptake through shaping health beliefs? Vaccine 37:1046-1052

20. Health Quality Ontario (2016) The reality of caring. Distress among the caregivers of home care patients. Health Quality Ontario

21. Thorpe JM, Sleath BL, Thorpe CTK et al (2006) Caregiver psychological distress as a barrier to influenza vaccination among community-dwelling elderly with dementia. Med Care 44:713-721

22. Abramson $\mathrm{ZH}$, Levi $\mathrm{O}$ (2008) Is performance of influenza vaccination in the elderly related to treating physician's self Immunization and other physician characteristics? Prev Med (Baltim) 47:550-553

23. Banach DB, Ornstein K, Factor SH et al (2012) Seasonal influenza vaccination among homebound elderly receiving home-based primary care in New York City. J Community Health 37:10-14

24. Bean SJ, Catania JA (2018) Immunology beliefs as a factor in vaccine opposition among complementary and alternative medical providers. SAGE Open Med 6:205031211880762

25. Shah SM, Carey IM, Harris T et al (2012) The impact of dementia on influenza vaccination uptake in community and care home residents. Age Ageing 41:64-69

26. Henkel PJ, Marvanova M (2018) Rural Disparities in Alzheimer's disease-related community pharmacy care in the United States. J Rural Heal 34:347-358

27. Smailbegovic MS, Laing GJ, Bedford H (2003) Why do parents decide against immunization? The effect of health beliefs and health professionals. Child Care Health Dev 29:303-311

28. A Voice for Choice (2018) No Title [Internet]. http://avoiceforc hoice.org. Accessed 20 Jan 2020

29. Evrony A, Caplan A (2017) The overlooked dangers of anti-vaccination groups' social media presence. Hum Vaccines Immunother 13:1475-1476

30. Davies P, Chapman S, Leask J (2002) Antivaccination activists on the world wide web. Arch Dis Child 87:22-25

31. Wolfe RM, Sharp LK, Lipsky MS (2002) Content and design attributes of antivaccination Web sites. J Am Med Assoc 287:3245-3248

Publisher's Note Springer Nature remains neutral with regard to jurisdictional claims in published maps and institutional affiliations. 